\title{
The Pareto managerial principle: when does it apply?
}

\author{
ABRAHAM GROSFELD-NIR $\dagger$, BOAZ RONEN*† and NIR KOZLOVSKY§ \\ $†$ Academic College of Tel-Aviv-Yaffo, Israel \\ $\Varangle$ Tel Aviv University, Faculty of Management, Israel \\ $\S$ Tel Aviv University, Faculty of Management, Israel
}

(Revision received March 2006)

\begin{abstract}
The well-known 80/20 Pareto principle and its refinement into "A", "B", and " $C$ " categories give rise to a managerial methodology consisting of three steps: classification; differentiation; and resource allocation. This is an easy-to-implement and extremely effective methodology. It starts with the creation of "Pareto diagrams", i.e. bar charts of attributes and their relative frequency, presented in descending order. Typically, Pareto diagrams are useful in that they provide managers with a summary of practical information, revealing critical attributes. However, sometimes a Pareto diagram is less informative than it might be, because the relative frequency is almost uniform. The objective of this article is to provide an analytical tool (an index) that employs the abovementioned methodology to measure the closeness of empirical Pareto diagrams to an "ideal" Pareto diagram. The index developed is based upon entropy.
\end{abstract}

Keywords: Pareto principle; Entropy; Theory of constraints

\section{Introduction}

The overwhelming flow of information forces busy managers to seek focusing methods that allow for efficient utilization of limited time resources. Among these methods are the well-known theory of constraint (TOC), which advocates the detection of critical resources (bottlenecks), and total quality management (TQM), which advises focusing on resources and activities imperative for quality. In this context, the Pareto methodology, described below, is one of the most important managerial tools that can be used to identify factors worthy of attention.

Early in the 20th century, the economist Vilfredo Pareto stated that $20 \%$ of the population possesses $80 \%$ of the wealth. This basic $80 / 20$ principle was then adopted in describing other realities; and, later, enhanced to the "A", "B", "C" classification, in which the "A" group (the "vital few"), consisting of approximately $20 \%$ of the attributes (items), accounts for $80 \%$ of the phenomenon; the "B" group, i.e. the next $30 \%$ of the items, accounts for $10 \%$ of the phenomenon, and the " $\mathrm{C}$ " group (the "trivial many"), which contains $50 \%$ of the items, accounts for only $10 \%$ of the phenomenon.

*Corresponding author. Email: boazr@post.tau.ac.il 
Many researchers find it useful to model managerial scenarios according to the Pareto principle: approximately $20 \%$ of the projects generate $80 \%$ of the revenues (or profits); $20 \%$ of the customers generate $80 \%$ of a firm's earnings; $20 \%$ of the part numbers in a given bill of materials (BOM) account for $80 \%$ of the value of all parts. The same goes for stock keeping units (SKUs) at the supermarket and so on. Koch (1998) presents examples in many areas of management, including business, marketing, finance, human resources, production and logistics.

Ronen et al. (2005) suggested and explored a "Pareto focusing methodology" that consists of three steps:

- Classification - sort out symptoms (attributes) of a phenomenon to build a Pareto diagram; use the diagram to identify the three classes "A", "B" and "C".

- Differentiation - set a specialized policy for each class.

- Allocation - assign resources accordingly.

Typically, a useful or ideal Pareto chart (figure 1) is one where about $20 \%$ of the attributes have an $80 \%$ weight in terms of relative frequency, thus revealing critical features. However, sometimes, a Pareto chart is less informative, because the different attributes occur with almost uniform relative frequency (figure 2).

While some empirical Pareto charts are easy to classify as useful or useless (almost uniform), many others are in between and it is not absolutely obvious whether the three-step Pareto focusing methodology applies. The objective of this article is to provide an analytical tool (an index) which advocates the use of the methodology to measure the closeness of empirical Pareto charts to an "ideal" Pareto chart. The index developed is based upon entropy.

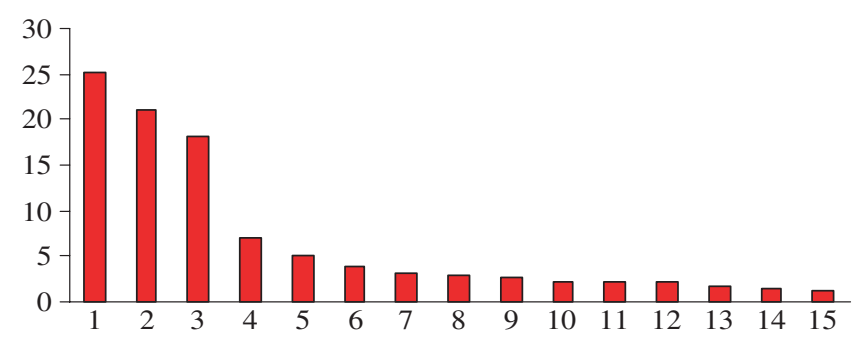

Figure 1. A differentiating Pareto diagram.

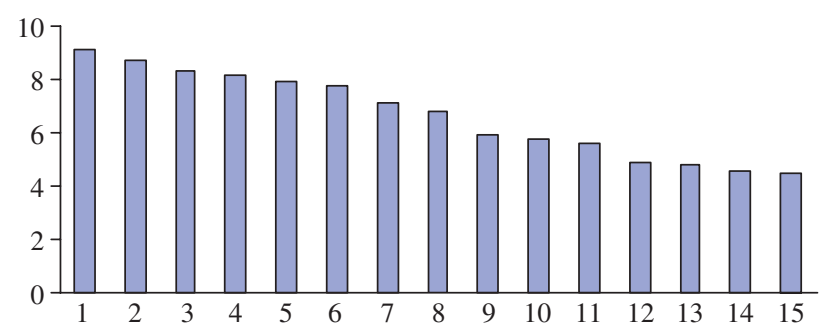

Figure 2. An almost uniform Pareto diagram. 
Section 2 of the paper presents the literature and research carried out. Section 3 describes the motivation for developing the tool. Section 4 develops the entropybased index to measure the closeness of a given Pareto chart to an ideal chart. Section 5 demonstrates the application of the model. Section 5 summarizes and concludes the paper.

\section{Literature}

Many managerial tools are useful in that they reveal attributes worthy of attention. Such as, for example, the TOC, advocating a focus on critical resources (bottlenecks); TQM, advocating a focus on resources and activities imperative for quality; and the "complete kit", advocating starting a job only after all the required parts are available. For literature describing the TOC, see Goldratt (1990); Ronen and Spector (1992); and Mabin and Balderstone (2000). The literature relating to TQM is vast: e.g. Deming (1985) and Juran (1988). The "complete kit principle" is described in Ronen (1992) and Grosfeld-Nir and Ronen (1998). In this context the Pareto principle, coupled with Pareto charts, is an extremely useful and easy-toimplement tool that reveals attributes worthy of attention.

Despite its high potential value and many applications, the Pareto principle has been the subject of few research and academic studies. Herbert (1995) suggested the use of the principle in managing storage houses: the use of Pareto charts allowed focusing on stored items and placing them in the warehouse in the most optimal way. Gleason (1995) applied the Pareto principle to the paper mill industry, using Pareto charts to identify frequent failures in order to increase machine utility. Indeed, the America-Israel Paper Mills used Pareto charts successfully for several years as a tool to increase productivity of high-cost resources. The approach was labeled "lost time analysis" (LTA) (Gleason 1995). A banking application of the Pareto principle was employed by Hales (1995) to locate and develop profitable sectors for targeting marketing efforts. An information systems research study using the Pareto principle was conducted and reported by Ronen and Spiegler (1991), where the methodological analysis performed on an insurance database demonstrated that $20 \%$ of the data are used to generate over $80 \%$ of the information accessed. In an age of exponentially growing databases, this finding has a significant effect on the design of databases and storage technology.

Temme (1994) established prioritization rules taking Pareto as the first and foremost among them. He suggested that the Pareto principle be employed to set priorities for focusing on the topics requiring most management attention. An interesting case study describing a successful and insightful application of Pareto in a large industrial firm is reported by Tatikonda et al. (1999). In this study, the firm decided to build a pricing system based on the ABC activities. At the start of this long and expensive project, five senior workers in the accounting department were sent to a seminar on the subject. Then, a steering committee of 15 workers from the various departments was established. After 2 years, in which thousands of man-hours and direct software, with a cost of $\$ 200000$, were invested, the first conclusions were formulated, the main one indicating that low-volume products generate higher indirect costs than high volume products. But, as the ABC process matured, the firm was acquired by a larger enterprise, and the new management 
immediately stopped the project. Instead, it decided on a series of steps all stemming from the Pareto principle: focusing on the $20 \%$ of the items that contribute $80 \%$ of the yield, cutting the number of suppliers and reducing the number of purchased raw materials. These steps, in addition to a "just in time" (JIT) implementation and quality-management methods led to significant achievements in many areas. Firm overheads diminished and various coordination and control departments were eliminated. The purchasing department, for example, which had previously employed 28 workers ended up with only three workers.

A model that analyses when the Pareto rule applies was not found in the literature.

\section{Pareto charts}

Statistically, a Pareto chart is simply a frequency block diagram displaying the relative frequency of different attributes in descending order. This classification is an essential step that must precede taking the corrective measures of differentiation and allocation.

For example, suppose that while studying a certain factor, such as the number of defectives, classification reveals 15 attributes. Thus, according to the Pareto principle, approximately $20 \%$, i.e. three attributes, are the principle source of $80 \%$ of the defectives, and focusing on improving these three attributes may eliminate most of the defectives.

It should be noted that the creation of a Pareto chart, i.e. classification, is usually a quick and inexpensive information gathering action. It is the implementation of the next two steps that requires a relative lengthy organizational effort. However, as the selection of the attributes which are candidates for corrective measures depends upon the outcomes presented in the Pareto chart, the information gathering effort needs special care, as mistakes in the classification may lead to irrelevant correction steps.

Although many researchers have reported applications where Pareto charts reveal that the $80 / 20$ principle holds true, sometimes, in practice, certain classifications produce Pareto charts which are less managerially helpful in that the relative frequency corresponding to $20 \%$ of the attributes is much less than $80 \%$, leading to a situation where focusing on a small number of attributes is impossible. Also, after obtaining a Pareto chart, managers sometimes find it hard to reach agreement about how useful the chart is in identifying a small number of critical attributes, that is, how closely the diagram fits the $80 / 20$ principle. For this reason we develop a quantitative tool, followed by a simple index, to measure the closeness of Pareto charts to the $80 / 20$ principle.

Taking a slightly broader point of view, a manager observing a Pareto chart may wonder how useful this particular chart is in guiding him to focus on a small number of attributes which are a major source of problems. For example, table 1 and figure 1 describe a situation where, intuitively, it would be desirable to focus on the first three attributes $(20 \%)$. In contrast, figure 2 displays a reality where the Pareto chart is almost uniform and, therefore, the Pareto principle should not be used.

Our goal is to provide a numerical index which will indicate that, for example, the attributes of figure 1 fit the three-step Pareto methodology while the attributes of figure 2 do not. Our approach is similar to the one used in statistics, where $95 \%$ is 
Table 1. Pareto table.

\begin{tabular}{lrrrrr}
\hline Attribute & $\%$ & Attribute & $\%$ & Attribute & $\%$ \\
\hline 1 & 25.2 & 6 & 3.9 & 11 & 2.1 \\
2 & 21.1 & 7 & 3.1 & 12 & 2.1 \\
3 & 18.2 & 8 & 2.9 & 13 & 1.6 \\
4 & 7.1 & 9 & 2.7 & 14 & 1.5 \\
5 & 5.2 & 10 & 2.1 & 15 & 1.2 \\
\hline
\end{tabular}

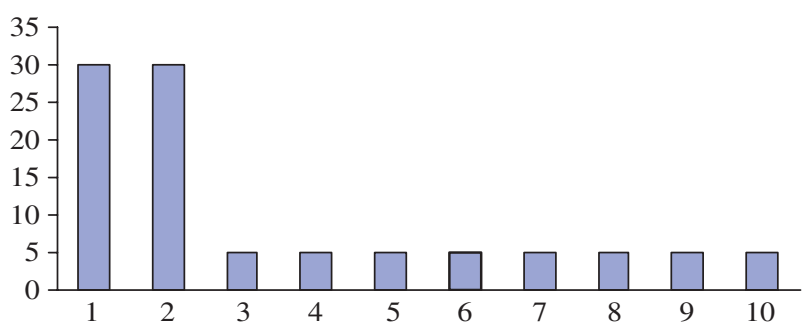

Figure 3. A Pareto control limit chart with 10 attributes.

used as a control limit to imply significance. Note that the $95 \%$ control limit is a convention based upon expert opinion. Similarly, based upon expert opinion we develop a Pareto index, with control limits, to indicate that a certain Pareto classification is "significant", i.e. fits the Pareto methodology.

\section{Pareto charts fitting the three-step methodology: using entropy}

Consider a Pareto chart with 10 attributes; then, clearly, if the relative frequency associated with the first two attributes is $80 \%$, or so, the Pareto differentiating methodology applies. However, if the relative frequency associated with the first two attributes is much less than $80 \%$, the Pareto differentiating methodology should not be used. After deliberation and consulting with experts we selected as a "control limit" a Pareto chart where $20 \%$ of the attributes represent a total of $60 \%$ of the relative frequency and the remaining attributes are uniform. For example, when there are 10 attributes, figure 3 represents the control limit chart.

To avoid confusion the relative frequencies of control limit charts for 5, 10, and 15 attributes are:

5 attributes : $60 \%, 10 \%, 10 \%, 10 \%, 10 \%$.

10 attributes : $30 \%, 30 \%$, and eight times $5 \%$.

15 attributes : $20 \%, 20 \%, 20 \%$, and 12 times $(40 / 12=) 3.33 \%$.

The entropy corresponding to 5 attributes is:

$$
\begin{aligned}
\mathrm{H}_{5} & =-0.6 * \ln 0.6-4 * 0.1 * \ln 0.1=1.77 \\
\mathrm{H}_{10} & =-2 * 0.3 * \ln 0.3-8 * 0.05 * \ln 0.05=2.77 \\
\mathrm{H}_{15} & =-3 * 0.2 * \ln 0.2-12 * 0.0333 * \ln 0.0333=3.35
\end{aligned}
$$


Table 2. Control limits based upon entropy.

\begin{tabular}{lllllllllll}
\hline No. of activities & 5 & 10 & 15 & 20 & 25 & 30 & 35 & 40 & 45 & 50 \\
Control limit & 1.77 & 2.77 & 3.55 & 3.77 & 4.09 & 4.36 & 4.58 & 4.77 & 4.94 & 5.09
\end{tabular}

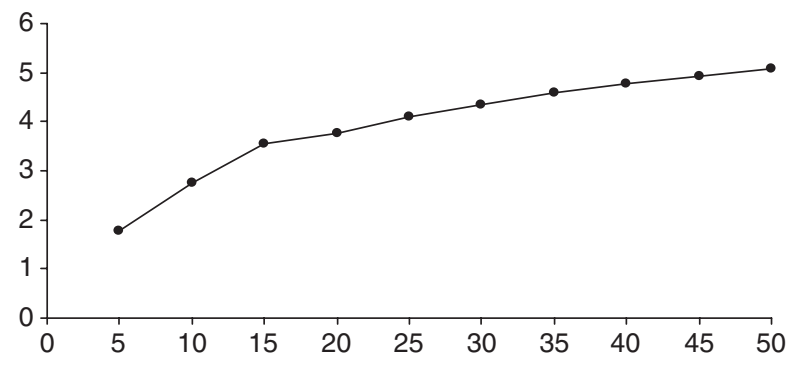

Figure 4. Control limits based upon entropy.

These numbers serve as control limits, namely, if, for example, a Pareto chart with 10 attributes has entropy exceeding the control limit 2.77, it should not be treated according to the Pareto methodology.

Table 2 and figure 4 display the control limits for Pareto charts with up to 50 attributes. If the number of activities is not a multiple of five, interpolations can be used.

For example, the entropy corresponding to figure 1 is $\mathrm{h}=3.16<3.35=\mathrm{H}_{15}$, which implies that the Pareto methodology is adequate. On the other hand, the entropy corresponding to figure 2 is $\mathrm{h}=3.87>3.35=\mathrm{H}_{15}$, which implies that the Pareto methodology should not be used.

\subsection{Rule of thumb}

Sometimes, observing the Pareto frequency chart, it is obvious that it is adequate for the use of the Pareto methodology, and the formal use of the entropy index becomes a burden. Mathematically, the following "rule of thumb" is a sufficient condition: if the total frequency corresponding to $20 \%$ of the attributes is above $60 \%$ the entropy index is below the control limit, i.e. the use of the Pareto methodology is in place.

\section{Applying the model}

We will now demonstrate the application of the model, using the three-step methodology. 


\subsection{Case I: a company in the aviation industry}

In 2002 the company described was manufacturing about 800 different high-tech products aimed at the aviation industry. However, in spite of an impressive monetary figure for total sales net profit was low. It has been suggested that the company needs to improve its focus in terms of product variety and marketing.

- Classification. First two methods of classification were used: (a) an overall classification of all products according to their monetary contribution; and (b) products were sorted into 14 group technologies; then groups were classified according to their monetary contribution.

The overall classification revealed that class "A", consisting of about 160 products $(20 \%)$, accounts for $78 \%$ of the contribution; class " $\mathrm{B}$ " consisting of about 240 products $(30 \%)$ accounts for about $14 \%$ of the contribution, and class "C" (the "trivial many"; $50 \%$ ) consisting of about 400 products, accounts for only $8 \%$ of the contribution. Moreover, almost all the products of class " $\mathrm{C}$ " belonged to six group technologies. Note that, using the "rule of thumb" (end of section 4) for the overall classification, the entropy index recommends the use of the Pareto methodology. Also, omitting details, calculating the entropy index for the group technologies, gave the same results.

- Differentiation: the company applied a different policy for each class. Management concluded that there were too many parts requiring marketing and logistic efforts with relatively low added monetary value. Further investigation revealed that low profitability for some products was expected as in the market there were competing low-price products. The company presented three different policies to the three part classes.

- Class "C": about 250 products were discontinued. This eliminated two group technologies, the residuals of which were merged with others. Discontinuation was carried out in two ways: for some parts, a notification stating the discontinuation of the parts was sent to the customers, allowing time to carry stocks; for other parts, a significant increase in price was announced, explaining the extra costs needed to continue holding the parts active. This policy intended many " $\mathrm{C}$ " parts to discontinue, while others move to the "B" class.

- Class B: use some of the available sales force to increase sales of selected items, trying to turn them into class " $\mathrm{A}$ " items. Other than that, basically, no change in activities.

- Class A: customers will get special attention; their needs will be learned and their satisfaction will be monitored in great detail. Marketing will make an effort to identify potential customers (currently buyers of competing products) and offer specials and quality service for selected products.

- Allocation: most of the sales resources were allocated to the "A" items, and the rest to the "C" items.

Within one year the Pareto chart moved to a more even shape, where about $30 \%$ of the parts had $60 \%$ of the contribution. The "fear" that many customers would 
quit unless they were offered a "full suite" proved to be ungrounded. Although total sales dropped, net profit increased by an impressive percentage.

\subsection{Case II: a supermarket chain}

In 2001, the supermarket chain described here owned 220 stores. Again, in spite of an impressive monetary figure for total sales, net profits were low. When a new management team came into office, they started to analyse the profitability of the chain via the stores. First, they analysed the contribution of each store and drew a Pareto chart. Then, they applied the Pareto methodology as follows:

- Classification: they classified the stores by their contribution into " $\mathrm{A}$ ", " $\mathrm{B}$ " and "C". As the "A" stores $(20 \%)$ contributed over $60 \%$ of the revenue it became clear that the entropy index was below the control limit, showing that there was a potential to carry on the analysis using the contribution per store as a leading variable.

- Differentiation: the "A" stores went through a retention program to keep the customers in the chain, and increase their contribution. The "B" stores went through a growth program that increased the contribution per store. The " $\mathrm{C}$ " stores were analysed: some of them were closed down (especially in residential areas that turned commercial).

- Resource allocation: most of the managerial attention was given to the "A" and "B" stores. Also, managers of "A" stores were asked to spend time at " $\mathrm{B}$ " and "C" stores to instigate changes in terms of managerial methods and product variety; and managers of " $\mathrm{B}$ " and " $\mathrm{C}$ " stores were asked to spend time at "A" stores to see if they could adopt methods and products.

At the same time management analysed the stores' achievements by analysing the contribution per dollar of rent of each store. This parameter enabled management to compare small and large stores with the same metric.

- Classification: the stores were classified into "A", "B", and "C" types. An index analysis showed a significant potential for using the Pareto methodology.

- Differentiation: the "A" stores were focusing on retention, while the "B" stores focused on growth. The "C" stores were candidates for management replacement, or closure.

- Resource allocation: most of the resources were allocated to the "A" and "B" stores.

- After 3 years of continuous improvement, an index analysis was carried out again. The figures revealed that these two parameters (contribution per store and contribution per dollar rent) were no longer significant.

\section{Conclusions}

Pareto charts, describing the relative frequency of a main factor as a function of attributes, make for an extremely useful managerial focusing tool. However, the charts must be differentiating, i.e. the relative frequency associated with a small 
number of attributes must be high enough. In this context, the presence of an analytical tool helping to identify useless charts is important.

Our index, based on entropy, combines common sense, i.e. expert opinion with a formal analytically calculated index. The resulting index provides a control limit that depends on the number of attributes. As we observed many classifications corresponding to this index, we concluded that it is useful and practical.

Future empirical research could consider the comparison of our index to judgmental expert opinion and report how well it works.

\section{References}

Deming, E.W., Transformation of western style management. Interfaces, 1985, 15, 6-11.

Gleason, M., High level of operating equipment efficiency requires overall effort. Pulp Pap., $1995,69,79-84$.

Goldratt, E.M., The Haystack Syndrome, 1990 (North River Press: Croton on Hudson, NY).

Grosfeld-Nir, A. and Ronen, B., The complete kit concept: modeling the managerial approach. Comput. Ind. Eng., 1998, 34, 695-701.

Hales, M.G., Focusing on 15\% of the pie. Bank Marketing, 1995, 27, 29-34.

Herbert, P.R., Separate the vital few from the trivial many. Transport. Dist., 1995, 36, 84-86.

Juran, J.M., Planning for Quality, 1988 (The Free Press/Macmillan: New York).

Koch, R., The 80/20 Principle, 1998 (Doubleday: New York).

Mabin, V.J. and Balderstone, S.J., The World of the Theory of Constraints, 2000 (The St. Lucie/APICS Series on Constraints Management: Boca Raton, FL).

Ronen, B., The "complete kit concept". Int. J. Prod. Res., 1992, 30, 2457-2466.

Ronen, B. and Spector, Y., Managing systems constraints: a cost utilization approach. Int. J. Prod. Res., 1992, 30, 2045-2061.

Ronen, B. and Spiegler, I., Information as inventory: a new conceptual view. Inform. Manage., 1991, 21, 239-247.

Ronen, B., Spiegler, I. and Kozlovsky, N., The use, misuse and abuse of the Pareto principle. Working paper, Tel Aviv University, Faculty of Management, 2005.

Tatikonda, L.U., O'Brien, D. and Tatikonda, R.J., Succeeding with 80/20. Manage. Account., 1999, 80, 40-44.

Temme, J., Learn to manage competing priorities by focusing on what is important. Plant Eng., 1994, 48, 80. 